\title{
Die Impfung gegen COVID-19 in Italien. Ethische und rechtliche Aspekte
}

Martin M. Lintner

Italien gehörte zu Beginn der Corona-Pandemie nicht nur in Europa, sondern weltweit zu den am stärksten von der Lungenkrankheit Covid-19 betroffenen Ländern. ${ }^{1}$ Bis zum 7. Juni 2021 wurden auf nationaler Ebene insgesamt 4.233.698 Infektionen und 126.588 Todesopfer gezählt ${ }^{2}$, was einer Todesrate von 2.093 pro Million Einwohner entspricht ${ }^{3}$. Die statistische Kurve zeigt drei Höhepunkte von täglichen Todesfällen auf: Ende März/Anfang April 2020 mit 13,47, Ende November/Anfang Dezember 2020 mit 12,26 und - bereits deutlich niedriger - Mitte April 2021 mit 7,89 Todesfällen pro Million Einwohner. Am 7. Juni 2021 wurden 65 Personen (1,07 pro Million Einwohner) verzeichnet, die am bzw. mit dem SARS$\mathrm{CoV}-2$-Virus verstorben sind.

\section{Die nationale Impfstrategie des Gesundheitsministeriums (1)}

Die wichtigste Maßnahme zur Überwindung der Covid-19-Pandemie stellt die nationale Impfstrategie dar. Die ersten Impfungen gegen das SARSCoV-2 wurden in Italien am 27. Dezember 2020 im römischen Krankenhaus Spallanzani verabreicht. Die gesetzliche Grundlage bildete das Dokument "Elemente zur Vorbereitung der Impfstrategie“, das vom italienischen Gesundheitsministerium am 2. Dezember 2020 vorgelegt und vom Parlament per Dekret am 2. Jänner 2021 erlassen worden ist. ${ }^{4}$ Zusammen

1 Siehe dazu Lintner: Das Triage-Problem in Italien während der COVID-19-Pandemie. Der vorliegende Artikel knüpft stilistisch und methodologisch an diesen Beitrag an, indem die Thematik vorwiegend dokumentarisch dargestellt und analysiert wird.

2 Vgl. Johns Hopkins University, COVID-19 Dashboard by the Center for Systems Science and Engineering (CSSE) [Stand: 09.06.2021].

3 Vgl. Our World in Data, Statistics and Research. Coronavirus (COVID-19) Deaths [Stand: 09.06.2021].

4 Ministero della Salute u. a., Vaccinazione Anti SARS-CoV-2/COVID-19. Piano strategico. 
mit den „Empfehlungen ad interim zu Zielgruppen für die Impfung gegen SARS-CoV-2/COVID-19“5 vom 10. März 2021, worin die zu impfenden Bevölkerungsgruppen und die Prioritäten aktualisiert wurden, bildete es die am 12. März 2021 per Dekret verabschiedete nationale Impfstrategie ${ }^{6}$, auf die weiter unten noch detaillierter eingegangen wird.

\section{Die Stellungnabmen des Nationalen Komitees für Bioethik}

\subsection{Stellungnahme über ethische Aspekte bezüglich der Erforschung, der Herstellung, der Kosten und der Verteilung von Covid-19-Impfstoffen (27.11.2020)}

Bereits am 27. November 2020 veröffentlichte das Nationale Komitee für Bioethik eine Stellungnahme über ethische Aspekte bezüglich Erforschung, Herstellung, Kosten und Verteilung von Covid-19-Impfstoffen. ${ }^{7}$ Dabei wird zunächst betont, dass aufgrund der damaligen noch ungewissen Kenntnisse über das Virus auf wissenschaftlicher und epidemiologischer Ebene die Erforschung und Herstellung von Impfstoffen mit besonderer Sorgfalt erfolgen müssen. Die weiterhin herrschende Notsituation dürfe nicht dazu führen, die auf wissenschaftlicher, bioethischer und biojuristischer Ebene nötigen Zeitabläufe für Untersuchungen und klinische Versuche zu umgehen, vielmehr müsse die Qualität der Studien und der Schutz der teilnehmenden Personen gewährleistet werden. Ein weiterer Aspekt der Stellungnahme betrifft die gerechte Verteilung der Impfstoffe auf nationaler und internationaler Ebene. Die Impfstoffe werden als Allgemeingut bezeichnet. Gefordert wird, dass die Pharmaunternehmen ihre soziale Verantwortung wahrnehmen und internationale Abkommen eine gerechte globale Verteilung der Impfstoffe garantieren. Thematisiert wird die Problematik von Patentrechten: Sie zu früh aufzuheben würde die Forschung und Entwicklung von Impfstoffen bremsen, andererseits können Patentrechte eine gerechte Verteilung des Impfstoffs behindern. Jedenfalls fordert das Komitee, dass die Politik Produktion und Vertrieb, die nicht allein durch Marktgesetze geregelt werden dürfen, steuern soll. Wie kaum je

5 Ministero della Salute u. a., Vaccinazione Anti SARS-CoV-2/COVID-19. Raccomandazioni ad interim.

6 Vgl. Ministero della Salute, Piano vaccini anti COVID-19.

7 Vgl. Comitato nazionale di Bioetica, I vaccini e COVID-19. 
zuvor habe die Erforschung und Herstellung von Covid-19-Impfstoffen nicht nur eine pharmazeutische, sondern auch eine politische Bedeutung:

Wer sie zuerst produziert, kann damit seine wissenschaftliche und technologische Kompetenz behaupten und demonstrieren in der Lage zu sein, zuerst die Bewohner der eigenen Nation und dann jene verbündeter Länder zu schützen. Der wirtschaftliche wird so auch zum politischen Wettbewerb und zum Maß von Macht. ${ }^{8}$

Gefordert werden schließlich auch ethische Kriterien zur Verteilung der Impfstoffe sowie eine Festlegung der Prioritäten bestimmter Personengruppen anhand von transparent kommunizierten Kriterien, die auf der Grundlage neuer wissenschaftlicher Erkenntnisse über den Impfstoff, der Menge der verfügbaren Dosen und der jeweils aktuellen Infektionslage je neu aktualisiert werden müssen. Mit Verweis auf die ethische Stellungnahme des Komitees über Triage in einer pandemischen Notsituation und bei klinischem Ressourcenmange ${ }^{9}$ werden als die drei grundlegenden Kriterien genannt:

der allgemeine moralische, deontologische und rechtliche Grundsatz der gleichen Würde jedes Menschen, die Vermeidung jeglicher Diskriminierung sowie der integrative Grundsatz der Gerechtigkeit, [...] der bei ungleichen Ausgangsbedingungen eine substanzielle Gleichheit unter Berücksichtigung der Schutzbedürftigkeit für spezifische Bedürfnisse garantiert ${ }^{10}$.

Grundsätzlich empfiehlt das Komitee folgende Priorisierung:

Menschen, die bei der Durchführung ihrer Arbeit direkt in Kontakt mit Covid-19-Patienten kommen, und zwar mit direkten und indirekten Risiken (Ärzte, Krankenschwestern, Gesundheitspersonal, Hilfspersonal, Krankenwagenfahrer, Forscher, etc.); Personen, die für die Aufrechterhaltung wesentlicher Dienste von öffentlichem Nutzen unentbehrlich und für die Aufrechterhaltung der öffentlichen Ordnung und der sozialen Funktionsfähigkeit notwendig sind (Armee, Polizei, Transportwesen, Lehrer, Lebensmittellieferanten, Unternehmer usw.); ältere Menschen sowie Erwachsene und Minderjährige mit schwerwiegenden Gefährdungen und mit einem erhöhten Risiko einer vorher-

8 Ebd., 6 (Übersetzung hier und folgend durch den Verf.).

9 Vgl. Comitato nazionale di Bioetica, COVID-19 - La decisione clinica in condizioni di carenza di risorse e il criterio del "triage in emergenza pandemica“.

10 Comitato nazionale di Bioetica, I vaccini e COVID-19, 9. 
sehbaren Verschlechterung ihres Gesundheitszustands im Falle einer SARS-Cov-2-Infektion. ${ }^{11}$

Das Komitee erachtet es als notwendig, dass alle Anstrengungen unternommen werden, um eine optimale Durchimpfungsrate von 60-70 Prozent der Bevölkerung zu erreichen und aufrechtzuerhalten. Ausdrücklich betont wird das Prinzip der Freiwilligkeit der Impfung sowie der informierten Zustimmung bzw. des Abwehrrechts. Um die Impfbereitschaft $\mathrm{zu}$ fördern, wird eine gezielte transparente und multidisziplinäre Kommunikationsstrategie eingemahnt, die auf Evidenzen und aktuellen wissenschaftlichen Fakten basiert und zugleich Fehl- und Desinformationen entgegentritt. Für den Fall einer anhaltenden pandemischen Notsituation wird, mit Verweis auf die negativen sozialen und wirtschaftlichen Folgen von langfristigen Maßnahmen zur Eindämmung der Pandemie einerseits und auf den individuellen wie kollektiven gesundheitlichen Schutz andererseits, eine Impfplicht für Berufsgruppen, die einem erhöhten Risiko der Infektion und Übertragung von Viren ausgesetzt sind, ausdrücklich empfohlen. Die Impfpflicht dürfe jedoch nur so lange gelten wie die pandemische Notsituation anhält. ${ }^{12}$

\subsection{Motion über die Dringlichkeit der Impfung (12. März 2021)}

Am gleichen Tag, an dem das Parlament die „Empfehlungen ad interim zu Zielgruppen für die Impfung gegen SARS-CoV-2/COVID-19“ als Dekret erlassen hat, meldete sich das nationale Bioethikkomitee mit einer Motion über bioethische Aspekte der Dringlichkeit der Covid-19-Impfung zu Wort. ${ }^{13}$ Betont wird erneut das ethische Erfordernis, dass die nationale Impfstrategie die Zugangsgerechtigkeit garantiert und Ungleichheiten bei der Verteilung von Impfstoffen vermeidet, mit besonderem Augenmerk auf diejenigen, die aus medizinischen und sozialen Gründen besonders gefährdet sind (ältere oder kranke Menschen mit schwerer Gebrechlichkeit, Menschen in wirtschaftlich-sozialer Notlage). Die entsprechende Kriteriologie müsse auf nationaler Ebene nach einheitlichen und eindeutigen Kriterien angewandt werden, um Gerechtigkeit und Fairness in den verschiedenen Regionen zu gewährleisten. Eingemahnt wird zudem eine höhere

11 Comitato nazionale di Bioetica, I vaccini e COVID-19, 10, Fußnote 18.

12 Ebd., 11-12.

13 Vgl. Comitato nazionale di Bioetica, Mozione - Urgenza vaccinale, aspetti bioetici. 
Effizienz und bessere Organisation der Verteilung der Impfstoffe, die zu langsam und in den verschiedenen Regionen zu unterschiedlich erfolge. Die Gerechtigkeit verlange auch, dass auf nationaler Ebene die pharmazeutischen Ressourcen zur Produktion und zum Vertrieb von Covid-19-Impfstoffen erhöht werden. Schließlich betont das Komitee die Dringlichkeit, unter Wahrung der Privatsphäre der Bürger und Bürgerinnen, all jene Daten zu veröffentlichen, die zur Erfassung des Standes der Pandemie sowie zu ihrer Überwindung notwendig sind.

Erwähnenswert ist zudem die Fußnote, in der das Komitee auf die wichtige Rolle der täglichen Information durch die Massenmedien hinweist. Es kündigt an, zu diesem Thema eine eigene Stellungnahme zu erarbeiten. ${ }^{14}$ Dies spiegelt die auch in Italien grassierende Problematik von Desund Falschinformationen nicht nur zum Corona-Virus, sondern besonders auch über die Herstellung und Wirkung der Impfstoffe gegen Covid-19 wider.

\subsection{Stellungnahme zum, Covid-19-Impfpass}

Am 30. April 2021 veröffentlichte das Komitee eine Stellungnahme zum geplanten Covid-19-Impfpass, d. h. eines Zertifikats zum Nachweis einer Impfung, einer Genesung von Covid-19 oder eines Antigen- bzw. PCRTests. ${ }^{15}$ Begrüßt wird dessen Zielsetzung, nämlich so bald wie möglich die Freiheitseinschränkungen zu lockern und gleichzeitig die Infektionsrate einzudämmen, damit soziale, wirtschaftliche, kulturelle, religiöse und andere Aktivitäten wieder aufgenommen werden können. Kritisch wird auf die Gefahr hingewiesen, dass ein gemeinsames Zertifikat nicht genüge, um die unterschiedliche Aussagekraft von Impfung, Genesung oder eines Antigen- bzw. PCR-Tests in Bezug auf Effizienz und Dauer des Schutzes vor Ansteckung zu dokumentieren. Das Komitee gibt weiters mögliche soziale Spannungen hinsichtlich einer Ungleichbehandlung zwischen denen, die sich impfen lassen konnten, und denen, die sich trotz Impfwilligkeit aufgrund der Knappheit von Impfstoffen noch nicht impfen lassen konnten, zu bedenken, ebenso eine mögliche Heterogenität bei der Anwendung der Bescheinigungen und das psychologische Risiko eines falschen Sicherheits-

14 Vgl. ebd., 6, Fußnote 15. Bis Abschluss des vorliegenden Manuskripts wurde die Stellungnahme noch nicht veröffentlicht.

15 Comitato nazionale di Bioetica, Passaporto, patentino, green pass nell'ambito della pandemia Covid-19: aspetti bioetici. 
gefühls. Es wird auch die Gefahr thematisiert, dass es sich um einen Präzedenzfall für eine künftige dauerhafte Verwendung eines biologischen Passes handeln könnte. Als Vorteile des Zertifikats werden genannt: ein erfordertes Entgegenkommen gegenüber jenen, die sich in Mitverantwortung geimpft haben und die Risiken einer Impfung auf sich genommen haben; ein Anreiz, dass ,zögerliche' Impfwillige sich eher impfen lassen; eine größere Bewegungsfreiheit bei gleichzeitiger strikter Beachtung der Maßnahmen zum Schutz der öffentlichen Gesundheit. Solange nicht für alle Impfbereiten Impfstoffe zur Verfügung stehen, werden schließlich kostenlose serologische Tests oder Abstriche, eine umfassende und verständliche Informationskampagne über Möglichkeiten und Grenzen, eine Regelung durch ein Landesgesetz sowie die zeitliche Befristung dieser Maßnahme empfohlen. Eingemahnt wird der Schutz von spezifischen gesundheitsbezogenen Daten.

\section{Die vatikanische Position zu den COVID-19-Impfungen}

Nachdem bekannt geworden war, dass für die Herstellung (z. B. von AstraZeneca und Johnson \& Johnson) sowie bei der Testung (z. B. von Moderna und BioNTech-Pfizer) von Covid-19-Impfstoffen Zelllinien verwendet werden, die nach Abtreibungen aus embryonalen Zellen gewonnen worden sind ${ }^{16}$, wurden weltweit die ethische Zulässigkeit dieser Herstellung und die moralische Erlaubnis, solche Impfstoffe zu erhalten, diskutiert. Konkret handelt es sich um Zellen aus fetalem Gewebe, das aus Föten nach deren Abtreibung zwischen 1960 und 1985 isoliert und im Labor weitergezüchtet wurde. Zwei Zelllinien (WI-38 und MRC5) werden zur Herstellung von Impfstoffen aus lebenden, abgeschwächten Viren (d. h. Aktiv-Impfstoffen) verwendet, eine dritte Zelllinie (HEK-293) kommt in bestimmten Phasen der Entwicklung von einigen Impfstoffen zur Anwendung. ${ }^{17}$ Bereits seit den 1990er Jahren werden fetale Zelllinien bei der Her-

16 Vgl. Wadman, Abortion opponents protest COVID-19 vaccines' use of fetal cells; Prentice, COVID-19 Vaccine Candidates and Abortion-Derived Cell Lines; letztere Publikation auf der Webseite des Charlotte-Lozier-Instituts aktualisiert laufend die Liste der Impfstoffe und der Projektphasen, in denen embryonale Zelllinien verwendet werden.

17 Vgl. Kummer, Covid-19-Impfstoffe: Ethische Stellungnahme zu Fragen der Herstellung; IMABE, COVID-19-Impfstoffe: Vatikan hält Einsatz für „moralisch akzeptabel“. 
stellung von Impfstoffen gegen Masern-Mumps-Röteln, Tollwut, Windpocken und Hepatitis A verwendet.

\subsection{Stellungnahme der ,Päpstlichen Akademie für das Leben' zur Verwendung von embryonalen Zelllinien bei der Herstellung von Impfstoffen (2005)}

Die Frage nach der ethischen Beurteilung der Verwendung von Zelllinien aus embryonalen Zellen bei der Produktion von Impfstoffen wurde bereits vor vielen Jahren seitens katholischer Lebensschutzbewegungen auch an den Vatikan herangetragen. 2005 hat die Päpstliche Akademie für das Leben zum ersten Mal eine diesbezügliche Stellungnahme veröffentlicht. ${ }^{18}$ Die Akademie argumentiert, dass die Verwendung dieser Zelllinien aus ethischer Perspektive problematisch ist und dass daher die Pflicht besteht, nach alternativen Herstellungsmethoden zu suchen oder alternative Impfstoffe zu verwenden. Im Falle einer Notlage, in der aus zeitlichen Gründen alternative Herstellungsmethoden nicht möglich sind oder alternative Impfstoffe nicht zur Verfügung stehen, erachtet sie die Verwendung von embryonalen Zelllinien sowie den Gebrauch dieser Impfstoffe jedoch für ethisch zulässig. Betont wird allerdings, dass dies keine Zustimmung zur bereits erfolgten Abtreibung bedeutet. Entsprechend der traditionellen Lehre der cooperatio ad malum (Mitwirkung am Bösen bzw. an unrechten Handlungen $)^{19}$ wird daher differenziert zwischen der formalen Mitwirkung im Sinne von inhaltlicher Zustimmung und der materiellen Mitwirkung, die in diesem Fall als lediglich mittelbar und entfernt anzusehen sei, da die Abtreibungen zeitlich lange zurückliegen und keine unmittelbare Mitwirkung an der Abtreibung vorliegt. Bei mangelnden Alternativen ist die Verwendung bereits vorhandener embryonaler Zelllinien, für deren Gewinnung eine Abtreibung nicht eigens durchgeführt worden ist, ethisch zulässig, sofern dies keine inhaltliche Zustimmung zur Abtreibung beinhaltet. Abschließend betont die Akademie, dass die Verweigerung eines solchen Impfstoffes aus Gewissensgründen zu respektieren sei.

18 Vgl. Päpstliche Akademie für das Leben, Moralische Überlegungen zu Impfstoffen, für deren Produktion Zellen von abgetriebenen Föten verwendet werden.

19 Siehe dazu: Rosenberger/Schaupp (Hg.), Ein Pakt mit dem Bösen? 


\subsection{Die Positionierung der Kongregation für die Glaubenslehre (2008)}

Auf die Thematik ging auch die Glaubenskongregation in einer Instruktion über einige Fragen der Bioethik aus dem Jahr 2008 ein. ${ }^{20}$ Sie argumentiert zusätzlich zum bereits genannten Prinzip der cooperatio ad malum auch mit den zu berücksichtigenden „differenzierten Verantwortlichkeiten" ${ }^{21}$. Eltern sind verantwortlich dafür, ihre Kinder aus gesundheitlichen Gründen impfen zu lassen, während jene, die die Impfstoffe herstellen, verantwortlich sind, sie auf ethisch akzeptable Weise herzustellen. Auch bei der Produktion tragen jene, die keine Entscheidungsvollmacht haben, nicht dieselbe Verantwortung wie jene, die über die Ausrichtung und Methoden der Produktion entscheiden.

\subsection{Die Stellungnahme der,Päpstlichen Akademie für das Leben' zur Impfpflicht (2017)}

2017 veröffentlichte die Päpstliche Akademie für das Leben ein weiteres Dokument zu den Kinderimpfungen in Italien. ${ }^{22}$ Darin werden einerseits die Impfungen als medizinische Errungenschaften gewürdigt und andererseits die abnehmende Impfungsrate in Italien, besonders gegen Masern und Röteln, beklagt. Erneut wird auf die Problematik eingegangen, dass bei Impfstoffen gegen Röteln, Windpocken, Kinderlähmung und Hepatitis A embryonale Zelllinien verwendet werden, die jedoch nicht aus aktuellen Abtreibungen gewonnen werden. Die verwendeten Zelllinien seien „sehr weit von den ursprünglichen Abtreibungen entfernt und implizieren nicht mehr jene Verbindung der moralischen Kooperation, die für eine ethisch negative Bewertung ihrer Verwendung unerlässlich “23 sei. Trotz der grundsätzlichen ethischen Verpflichtung zur Herstellung von Impfstoffen ohne Verwendung von Stoffen abortiven Ursprungs, bekräftigt die Akademie aufgrund der fehlenden moralisch relevanten Kooperation mit den früheren Abtreibungen die „sittliche Verantwortung für das Impfen, um keine ernsthaften Gesundheitsrisiken für Kinder und die Bevölkerung insgesamt einzugehen" 24 .

20 Vgl. Kongregation für die Glaubenslehre, Instruktion Dignitas Personae über einige Fragen der Bioethik (08.12.2008).

21 Vgl. ebd., Nr. 34-35.

22 Vgl. Pontificia Accademia per la Vita, Nota circa l'uso dei vaccini.

23 Ebd.

24 Ebd. 


\subsection{Die Stellungnahme der Kongregation für die Glaubenslehre zur Herstellung und Verwendung von Covid-19-Impfstoffen (2020)}

Im Zusammenhang mit der Herstellung und Verabreichung von Covid-19-Impfstoffen veröffentlichte die Glaubenskongregation am 21. Dezember 2020 eine entsprechende Note ${ }^{25}$ - mutmaßlich wiederum als Reaktion auf Anfragen US-amerikanischer Lebensschutzgruppen, die den Vatikan dazu drängen wollten, die bisherigen Stellungnahmen zu revidieren. Entgegen dieser Erwartungen bekräftigte der Vatikan seine Position. Wiederholt werden die Argumente der ethisch relevanten Differenzierung unterschiedlicher Formen der Mitwirkung sowie unterschiedlicher Verantwortlichkeiten. Unbeschadet der Verpflichtung, „ethisch vertretbare Impfstoffe herzustellen, zu genehmigen, zu verteilen und anzubieten, die weder dem medizinischen Personal noch den zu Impfenden selbst Gewissensprobleme verursachen“26, ist es - so die Glaubenskongregation - „sittlich erlaubt, Impfungen gegen Covid-19 zu empfangen, die in ihrer Entwicklung und Herstellung Zelllinien von abgetriebenen Föten verwendet haben“27. Die Glaubenskongregation geht schließlich auch auf die Frage der ethischen Pflicht zur Impfung ein. Sie betont, dass „die Sittlichkeit der Impfung jedenfalls nicht nur von der Pflicht zur Bewahrung der eigenen Gesundheit abhängt, sondern auch von der Pflicht, das Gemeinwohl zu verfolgen" 28 . Auch wenn

in der Regel die Impfung keine moralische Pflicht darstellt [...], kann die Impfung empfohlen sein, vor allem, um die Schwächsten und am meisten Gefährdeten zu schützen. Jene aber, die aus Gewissensgründen Impfstoffe, die aus von abgetriebenen Föten stammenden Zelllinien hergestellt worden sind, ablehnen, müssen sich bemühen, durch andere prophylaktische Mittel und angemessenes Verhalten zu vermeiden, dass sie selbst Überträger des ansteckenden Erregers werden. Insbesondere müssen sie jegliches Risiko für die Gesundheit jener vermeiden, die aus medizinischen oder anderen Gründen nicht geimpft werden können und am gefährdetsten sind. ${ }^{29}$

25 Vgl. Kongregation für die Glaubenslehre, Note über die Moralität des Gebrauchs einiger Impfungen gegen Covid-19 (21.12.2020).

26 Ebd., Nr. 4.

27 Ebd., Nr. 2.

28 Ebd., Nr. 5.

29 Ebd. 
Trotz dieser Klarstellungen seitens des Vatikans wurde von Impfgegnern weiterhin behauptet, der Gebrauch von Impfstoffen, für deren Herstellung oder Testung abortive Zelllinien verwendet werden, seie aus ethischer Sicht grundsätzlich abzulehnen und würde der katholischen Lehre widersprechen. In den sozialen Medien kursierten sogar Videos, in denen behauptet wurde, Papst Franziskus, der zur Impfung aufgerufen hat und sich auch selbst hat impfen lassen, habe sich die Tatstrafe der Exkommunikation, d. h. des Ausschlusses aus der kirchlichen Gemeinschaft, zugezogen. ${ }^{30}$

\section{Die ethische Stellungnabme der Arbeitsgruppe des ISS bei der Testung von Covid-19-Impfstoffen}

Im Februar 2021 veröffentlichte eine Arbeitsgruppe des Höheren Instituts für Gesundheit (Istituto Superiore di Sanità - ISS) des nationalen Gesundheitsdienstes eine ethische Stellungnahme zur klinischen Testung von Covid-19-Impfstoffen. ${ }^{31}$ Anlass der Stellungnahme bildete der Umstand, dass aufgrund der dringlichen Nachfrage nach einer großflächigen Verfügbarkeit von Impfstoffen der Zeitrahmen von klinischen Studien verkürzt worden war. Die Arbeitsgruppe betonte die ethische Notwendigkeit, einerseits Verzögerungen bei den Genehmigungsverfahren zu vermeiden, andererseits aber die Anforderungen der wissenschaftlichen Methodik bei klinischen Studien nicht aufzugeben. Auch in einer pandemischen Notsituation, die einer sorgfältigen Abwägung zwischen beiden Aspekten bedarf, dürfen die allgemeinen ethischen und wissenschaftlichen Kriterien für klinische Studien nicht aufgeweicht werden. Als besonders kritisch wird der Einsatz von Placebos beurteilt, da Placebos generell nicht verwendet werden sollten, wenn ein bereits wirksames Produkt verfügbar ist.

Bereits im Dezember 2020 hatte der Virologe und Immunologe Roberto Burioni Covid-19-Impfstoff-Studien mit dem Einsatz von Placebos aus ethischer Sicht abgelehnt. ${ }^{32}$ Zwar verteidigte er grundsätzlich placebo-

30 Abtreibung gilt in der katholischen Kirche als vorsätzliche Tötung eines unschuldigen Menschen und somit als schwere sittliche Verfehlung, die mit der schwersten Strafe, dem Kirchenbann, belegt wird (vgl. Codex Iuris Canonici [1983], $\$ 1398$ ). Auch die formelle Mitwirkung, d. h. die Zustimmung oder Mitwirkung, wird mit dieser Strafe belegt (vgl. Katechismus der Katholischen Kirche [2003], Nr. 2272).

31 Vgl. Istituto Superiore di Sanità (ISS), Gruppo di lavoro "Bioetica COVID-19", Aspetti di etica nella sperimentazione di vaccini anti-COVID-19.

32 Vgl. Burioni, Coronavirus: l'efficacia del vaccino pone un dilemma etico. 
kontrollierte, doppelblinde klinische Studien zur Untersuchung der therapeutischen Wirksamkeit sowie von Neben- und Folgewirkungen von Medikamenten. Da erste Studien jedoch eine hohe Wirksamkeit der Covid-19Impfstoffe erwiesen hätten, würde dies bedeuten, dass die ,Placebo-Geimpften' für den Zeitraum der Studie dem Risiko einer Infektion ausgesetzt werden, die mit einer richtigen Impfung verhindert werden könnte. Der Verzicht auf ,Schein-Impfungen` wiederum würde die längerfristigen Studien über die Wirksamkeit und Sicherheit der Impfstoffe beeinträchtigen. Burioni regte eine gesellschaftliche Debatte über diese Frage an: Personen nicht impfen und sie einem nicht unerheblichen persönlichen Risiko aussetzen, um mehr Informationen über die Sicherheit dieser neuen Impfstoffe zu erhalten, oder sie impfen, um ihre Gesundheit zu schützen, während man auf die Informationen über die Sicherheit der Impfstoffe verzichtet? Dies sei - im Unterschied zur öffentlich breit diskutierten Frage, ob die Bürger das Recht haben, sich aus Unwissenheit und Egoismus nicht zu impfen und sich selbst und die gesamte Gemeinschaft in Gefahr zu bringen - eine ernsthafte Frage.

Als ethisch nicht akzeptabel lehnt die Arbeitsgruppe des ISS bei den klinischen Versuchen von Covid-19-Impfstoffen auch die sogenannten Challenge-Studien ab, d. h. absichtliche Infektionen von teilnehmenden gesunden Probanden, und zwar aufgrund des nicht vorhersehbaren, möglicherweise schweren bis tödlichen Verlaufs der Erkrankung. In Bezug auf die Durchführung und den Abschluss von klinischen Studien wird verlangt, dass sie in enger Korrelation mit dem Inzidenzfaktor der Infektionen organisiert werden sollen. Von einer schnellen bzw. kürzeren Studie kann nämlich eine größere Anzahl von Patienten profitieren, die kurzfristig ein hohes Risiko haben, sich zu infizieren, wobei das Risiko längerfristig aufgrund der zu erwartenden sinkenden epidemiologischen Kurve abnehmen wird. Die Arbeitsgruppe betonte zudem das ethische Erfordernis, die Bevölkerung in einfacher Sprache transparent und klar darüber zu informieren, was realistischerweise von den Impfstoffen in Bezug auf Wirksamkeit und Sicherheit erwartet werden kann, einschließlich möglicher Nebenwirkungen und unerwarteter Effekte. Ansonsten sei die Gefahr gegeben, dass bereits weit verbreitete Gefühle des Misstrauens geschürt und die Impfskepsis vergrößert werden. 


\section{Die Haltung zur Covid-19-Impfung in der Bevölkerung am Beispiel von Südtirol}

Am Beispiel von Südtirol, wo das Landesstatistikamt ASTAT im Jänner sowie im März-April 2021 zwei Stichprobenerhebungen mit geschichteter Wahrscheinlichkeitsstichprobe durchführte, soll die Einstellung der Bevölkerung zur Covid-19-Impfung aufgezeigt werden. ${ }^{33}$ In der Umfrage von März-April erklärte eine von sechs Personen, sich nicht impfen zu lassen, das entspricht ca. 16 Prozent der Gesamtbevölkerung. ${ }^{34}$ Als Gründe wurden angegeben (Mehrfachnennungen waren möglich):

- „Weil ich befürchte, dass ich durch die Impfung infiziert werde oder Nebenwirkungen auftreten“: 66 Prozent

- „Weil ich nicht an die Wirkung der Impfung glaube“: 51 Prozent

- „Weil ich den Behörden hinsichtlich meiner persönlichen Daten nicht vertraue": 13 Prozent

- „Weil ich glaube, dass die Impfung schmerzhaft ist“: 6 Prozent

- „Weil ich nicht an die Existenz von COVID-19 glaube“: 5 Prozent

57 Prozent der Befragten äußerten Bedenken in Bezug auf die Ungewissheit über Langzeitfolgen, 36 Prozent befürchteten mögliche soziale Probleme bei der Diskussion um Impfprioritäten, 22 Prozent hingegen waren der Überzeugung, dass die Impfkampagne lediglich dem Profit der Pharmaindustrie diene, 16 Prozent (also der Anteil an Personen, die sich nicht impfen lassen wollten) hielten die Impfungen für unwirksam und 15 Prozent der Befragten hielten sie für nicht notwendig, da sie darauf hofften, dass die Herdenimmunität auch auf natürlichem Wege erreicht werden kann. ${ }^{35}$

Das Erleben der Gefährdung durch die Pandemie hat sich auch auf die Einstellung zur Impfpflicht ausgewirkt: 19 Prozent gaben an, dass sie aufgrund der Erfahrung der Pandemie eine Impfplicht im Unterschied zu vorher mehr, 7 Prozent hingegen weniger befürworten würden. 74 Prozent antworteten, dass die Pandemie ihre Einstellung zur Impfpflicht nicht verändert habe. ${ }^{36}$

Untersucht wurden schließlich auch die Korrelationen mit psychologischen Eigenschaften bei Impfgegnern: „Vor allem ein verschwörerisches Weltbild senkt die Befürwortung von Impfungen. Die Angst vor der

33 Vgl. ASTAT, Covid-19 und Impfungen: Einstellungen und Verhalten der Bürger.

34 Vgl. ebd., 7-8.

35 Vgl. ebd., 8-9.

36 Vgl. ebd., 9. 
Krankheit hingegen erhöht sie. Andererseits reduziert das psychische Wohlbefinden einer Person die Angst, aber nicht die Impfbereitschaft, auch weil das Wohlbefinden den Glauben an Verschwörungstheorien verringert. “37

Altruismus und Compliance, d. h. Zustimmung zu den präventiven Maßnahmen gegen die Pandemie, hingegen förderten die Impfbereitschaft ebenso wie folgende soziodemographische Variablen: das Arbeiten im Gesundheitswesen, die Angst vor dem Virus, zunehmendes Alter. Folgende Variablen hingegen haben sich negativ auf die Impfbereitschaft ausgewirkt: der Glaube an Verschwörungstheorien; an Covid-19 erkrankt gewesen und genesen zu sein. Eine nur untergeordnete Rolle spielten hingegen Bildung, Studientitel, Muttersprache und politische Ausrichtung. ${ }^{38}$

\section{Die nationale Impfstrategie des Gesundheitsministeriums (2)}

Weiter oben wurde bereits die am 12. März 2021 per Dekret verabschiedete nationale Impfstrategie erwähnt. ${ }^{39}$ Der Impfplan sah vor, dass ab April 2021 täglich ca. eine halbe Million Menschen geimpft werden sollte. Um diesen Plan umzusetzen, wurden 300.000 Freiwillige benötigt. Als Ziel der Impfstrategie wurde die Verhinderung von Todesfällen durch Covid-19 und ein möglichst rasches Erreichen der Herdenimmunität gegen SARSCoV-2 angegeben. Die Priorisierung bzw. die Rangfolge berücksichtigten in erster Linie das Krankheitsrisiko, die Relevanz von Personen für das Funktionieren des politischen und gesellschaftlichen Lebens, die Impfstofftypen bzw. die jeweilige Altersfreigabe oder aber -beschränkung durch die Europäische Arzneimittelagentur sowie die Empfehlungen der italienischen Arzneimittelbehörde (die besonders bei AstraZeneca wiederholt modifiziert wurden) und die Impfstoff-Verfügbarkeit.

Die Rangfolge sah in einem ersten Moment folgende Personengruppen vor: Mitarbeiter des Gesundheits- und Sozialwesens sowohl im öffentlichen als auch im privaten Bereich; Bewohner und Personal von Altenpflegeeinrichtungen; Menschen im fortgeschrittenen Alter sowie mit Komorbiditäten und Behinderungen. Zu einem späteren Zeitpunkt wurden die Empfehlungen für die Priorisierung aktualisiert und in fünf Kategorien gegliedert. Kategorie 1 bildeten Menschen mit einem hohen Risiko einer An-

37 Ebd., 10.

38 Vgl. ebd., 10.

39 Vgl. Ministero della Salute, Piano vaccini anti COVID-19. 
fälligkeit für einen schweren bis tödlichen Verlauf einer Infektion durch SARS-CoV-2 (aufgrund von bestehenden Erkrankungen, Organschäden, beeinträchtigte Immunantwort etc.) sowie Schwerstbehinderte und mit ihnen zusammenlebende Familienangehörige und Betreuer und Betreuerinnen, die kontinuierliche Hilfe leisten. Die nächsten Kategorien wurden auf der Basis von Alterskriterien und dem Vorhandensein von Pathologien erstellt, um eine möglichst schnelle und faire Fortsetzung der Kampagne in allen Regionen zu ermöglichen: Personen im Alter von 70 bis 79 Jahren (Kategorie 2) sowie im Alter von 60 bis 69 Jahren (Kategorie 3); Menschen mit Komorbidität im Alter unter 60 Jahren (Kategorie 4) und schließlich der Rest der Bevölkerung im Alter von 16 bis 60 Jahren (Kategorie 5). Die Freigabe der Anmeldungen zu den Impfungen für die letzte Kategorie erfolgte italienweit am 3. Juni 2021, wenig später konnten in vielen Regionen auch Jugendliche zwischen 12 und 16 Jahren (in Südtirol beispielsweise am 11. Juni) zur Coronaschutzimpfung vorgemerkt werden. Unabhängig vom Alter wurden ab der ersten Kategorie auch Personen im Bildungsbereich (Schule, Universitäten), Polizei- und Einsatzkräfte und Gefängnispersonal priorisiert.

\section{Impfpflicht für Bedienstete im Gesundheits- und Pflegebereich}

Am 28. Mai 2021 billigte das italienische Parlament mit dem Gesetz 76/2021 40 eine Verordnung vom 1. April (Gesetzesentwurf 44/202141), die für das Gesundheits- und Pflegepersonal - Apotheker, Psychologen und Ärzte mit eigener Praxis inbegriffen - eine Impfpflicht vorsieht. Eine Dispensierung von der Impfpflicht kann ausschließlich bei einer nachweislichen gesundheitlichen Gefährdung durch eine Impfung beantragt werden, wobei betroffene Personen nicht mehr Aufgaben mit direktem Kontakt mit Patienten oder Kunden wahrnehmen dürfen. Ein Abänderungsantrag, die Impfpflicht auf das Gesundheitspersonal zu beschränken, das in direktem Kontakt zu Kranken steht, wurde abgelehnt. Italien ist somit die erste (und bis Verfassung dieses Manuskripts einzige) Nation, die die Pflichtimpfung gegen Covid-19 für Mitarbeiterinnen und Mitarbeiter im Gesundheitswesen und in sozio-sanitären Berufen eingeführt hat.

40 Online abrufbar unter: https://www.lavoro.gov.it/documenti-e-norme/normative/ Documents/2021/L-28052021-n-76.pdf [15.06.2021].

41 Online abrufbar unter: https://www.gazzettaufficiale.it/eli/id/2021/04/01/21G0005 6/sg [15.06.2021]. 
Bereits der Gesetzesentwurf hatte für heftige Debatten gesorgt ${ }^{42}$, nachdem angekündigt worden war, dass Impfverweigerer ("no vax“) vom Dienst suspendiert werden. In der Provinz Belluno gab es schon im März den Fall von zwei Krankenpflegern und acht Mitarbeitern eines Seniorenwohnheimes, die aufgrund der Weigerung, sich impfen zu lassen, vom Dienst suspendiert worden sind und deren Rekurs vom Arbeitsgericht abgelehnt worden ist. ${ }^{43} \mathrm{Da}$ sowohl das Dekret als auch das Gesetz zur Impfung weitgehend ohne Einbeziehung der betroffenen Bediensteten im Gesundheits- und Pflegebereich beschlossen worden sind, fühlten sich viele von ihnen übergangen und sprachen von einem „Impfzwang“, dem sie unterworfen würden. In den emotional geführten Debatten kam die Präzisierung, dass es sich - genaugenommen - weder um einen Zwang noch um eine Pflicht, sondern um ein Erfordernis zur Berufsausübung für sozio-sanitäre Tätigkeiten handelt, die dem bzw. der einzelnen Person die Entscheidungsfreiheit belässt, sich impfen zu lassen oder nicht, zu kurz.

Das Gesetz 76/2021 hat also schwerwiegende arbeitsrechtliche Folgen. Die vorläufig bis zum 31.12.2021 zeitlich befristete Regelung bestimmt, dass jene, die die Impfpflicht nicht akzeptieren, zumindest für die Dauer der zeitlichen Befristung vom Dienst suspendiert werden und für diesen Zeitraum keinen Gehalt erhalten. ${ }^{44}$ Die lokalen Sanitätsbetriebe schätzten Anfang Juni, dass italienweit ca. 20.000 „no vax“-Bedienstete im Gesundheits- und Pflegebereich von der Disziplinarmaßnahme betroffen sein werden. ${ }^{45}$

Das Gesetz hat auch ethische Implikationen, die insgesamt jedoch zu wenig thematisiert und diskutiert worden sind, auch wenn - wie weiter oben bereits erwähnt worden ist - das nationale Bioethikkomitee bereits im Dezember 2020 eine Impfpflicht im Gesundheitswesen für ethisch gerechtfertigt angesehen hat. ${ }^{46}$ Kritiker dieser Maßnahme machten hingegen darauf aufmerksam, dass das Personal im Gesundheits- und Pflegebereich seit Ausbruch der Pandemie bereits bis an die Grenzen seiner Kräfte und

42 Vgl. z. B. Marini, L'obbligo vaccinale per professionisti e operatori sanitari, n. 44/2021.

43 Vgl. Miscione, Conseguenze del rifiuto di vaccinazione anti Covid-19 dopo il D.L. 44/2021.

44 Vgl. Frassy, Considerazioni etiche e giuridiche sull'obbligatorietà dei vaccini anti Covid-19.

45 Poletto, Le Asl pronte a sospendere dal lavoro i 20 mila medici e infermieri No Vax, ma gli ospedali temono il caos.

46 Die folgenden ethischen Anfragen an die Impfpflicht verdanke ich dem Austausch mit dem Südtiroler Arzt und Landtagsabgeordneten Franz Ploner. 
darüber hinaus im Einsatz sei und einen unverzichtbaren Beitrag geleistet habe, die pandemische Notsituation zu bewältigen, jetzt aber zusätzlich dem Druck ausgesetzt werde, innerhalb kurzer Zeit eine Entscheidung zu treffen oder aber in Kauf zu nehmen, von der Arbeit suspendiert zu werden. Anstatt eine Impfpflicht zu beschließen, solle man vielmehr Überzeugungsarbeit leisten, um möglichst viele zu einer freiwilligen Impfung zu motivieren und somit sowohl das Selbstbestimmungsrecht als auch das Nichtschadensprinzip zu respektieren. Das innerhalb kurzer Zeit mehrfache Hin und Her bei der Zulassung und Altersbeschränkung der Vektorimpfstoffe von AstraZeneca und Johnson \& Johnson, d. h. die zeitweise Aussetzung sowie die teils gegenläufigen Altersbeschränkungen, hat für große Unsicherheit gesorgt, besonders bei jüngeren und Frauen mittleren Alters unter 55 Jahren, also jener Personengruppe, in der es nach einer Impfung mit AstraZeneca häufiger als bei anderen Gruppen zu Sinus- und Hirnvenenthrombosen gekommen ist. Ebenso wurde argumentiert, dass eine Impfung keine ,sterile', sondern lediglich eine klinische Immunität bewirke, d. h., dass geimpfte Personen weiterhin sowohl sich selbst infizieren als auch ansteckend sein können. Das erklärte Ziel, Herdenimmunität zu erreichen, bedeute zudem in erster Linie einen kollektiven, jedoch keinen individuellen Schutz. Auch sei aufgrund des (bei fehlenden Maßnahmen) unbekannten Reproduktionsfaktors des SARS-CoV-2 weiterhin ungewiss, wie hoch die Immunisierungsrate sein muss, um Herdenimmunität zu erreichen. Aufgrund dieser und weiterer offenen Fragen sei die Impfpflicht für Bedienstete im sozio-sanitären Bereich aus ethischer Sicht zu hinterfragen. Doch eine öffentliche Debatte darüber blieb weitgehend aus. ${ }^{47}$

\section{Ausblick}

Bis zum 7. Juni 2021 wurden italienweit 38.761.223 Impfdosen verabreicht. Insgesamt erhielten 13.190.876 Personen über 12 Jahren (das entspricht 24,31 Prozent der Gesamtbevölkerung) zwei Dosen und haben somit den Impfzyklus abgeschlossen.

Während der Fertigstellung dieses Manuskripts konnte im gesamten italienischen Staatsgebiet ein Nachlassen der täglichen Impfzahlen beobach-

47 Nachtrag: Bis Ende August erhielten 71,49 Prozent der über 12-Jährigen die abgeschlossene Impfserie. Der italienische Ministerpräsident Mario Draghi kündigte am 2. September für den Fall, dass bis Monatsende nicht die Impfquote von 80 Prozent erreicht wird, die Einführung einer generellen Impfpflicht an. 
tet werden. ${ }^{48}$ Mögliche Faktoren sind die Verunsicherung nach dem Tod einer 18-jährigen Patientin am 9. Juni 2021, die eine Woche zuvor mit AstraZeneca geimpft worden war $^{49}$ und deren Schicksal italienweit für großes Aufsehen und Anteilnahme gesorgt hatte, und die darauffolgende Einschränkung dieses Impfstoffs auf Personen über 60 Jahren, aber auch die sinkenden Inzidenzzahlen. Die Regierung hat deshalb neue Informationskampagnen angekündigt. Besonders im Gesundheitswesen will man durch mehr Informationen die Impfwilligkeit fördern und damit Entlassungen bzw. als deren Folge einen Personalnotstand verhindern. Es bleibt abzuwarten, ob die Regierung ihr erklärtes Ziel, bis Ende September 2021 80 Prozent der Bevölkerung zu impfen, erreichen wird.

\section{Literatur}

ASTAT: Covid-19 und Impfungen: Einstellungen und Verhalten der Bürger (28.05.2021), in: https://astat.provinz.bz.it/de/aktuelles-publikationen-info.as p?news_action=3008news_image_id=1110165 [15.06.2021].

Burioni, Roberto: Coronavirus: l'efficacia del vaccino pone un dilemma etico (11.12.2020), in: https:/www.medicalfacts.it/2020/12/11/coronavirus-lefficac ia-del-vaccino-pone-un-dilemma-etico/ [10.06.2021].

Comitato nazionale di Bioetica: COVID-19 - La decisione clinica in condizioni di carenza di risorse e il criterio del „triage in emergenza pandemica“ (08.04.2020), in: http://bioetica.governo.it/media/3987/p136_2020_covid-19-la-decisione-clinic a-in-condizioni-di-carenza-di-risorse-e-il-criterio-del-triage-in-emergenza-pandemi ca.pdf [07.05.2020].

Comitato nazionale di Bioetica: I vaccini e COVID-19: aspetti etici per la ricerca, il costo e la distribuzione (27.11.2020), in: http://bioetica.governo.it/media/4115/p 140_2020_vaccini-e-covid19_it.pdf [10.06.2021].

Comitato nazionale di Bioetica: Mozione - Urgenza vaccinale, aspetti bioetici (12.03.3021), in: http://bioetica.governo.it/media/4133/m25_2021_urgenza-vacci nale-aspetti-bioetici.pdf [10.06.2021].

Comitato nazionale di Bioetica: Passaporto, patentino, green pass nell'ambito della pandemia Covid-19: aspetti bioetici (30.04.2021), in: http://bioetica.governo.it/it /pareri/pareri-e-risposte/passaporto-patentino-green-pass-nell-ambito-della-pande mia-covid-19-aspetti-bioetici/ [16.06.2021].

48 Vgl. die regelmäßig aktualisierten Angaben https:/www.rainews.it/ran24/speciali/ 2020/covid19/vaccini.php [17.06.2021].

49 Die Verstorbene litt an der Autoimmunerkrankung Thrombozytopenie und wurde medikamentös und hormonell behandelt. 
Frassy, Dario: Considerazioni etiche e giuridiche sull'obbligatorietà dei vaccini anti Covid-19 (07.06.2021), in: https:/www.altalex.com/documents/news/2021/06/0 7/considerazioni-etiche-e-giuridiche-sull-obbligatorieta-dei-vaccini-anti-covid-19 [15.06.2021].

IMABE: COVID-19-Impfstoffe: Vatikan hält Einsatz für „moralisch akzeptabel“ (17.01.2021), in: https://www.imabe.org/bioethikaktuell/einzelansicht/covid-19-i mpfstoffe-vatikan-haelt-einsatz-fuer-moralisch-akzeptabel [10.06.2021].

Istituto Superiore di Sanità (ISS), Gruppo di lavoro "Bioetica COVID-19": Aspetti di etica nella sperimentazione di vaccini anti-COVID-19 (18.02.2021), in: https:// www.iss.it/documents/20126/0/Rapporto+ISS+COVID-19+3_2021.pdf/00fc6054 -ded3-5de8-d32f-59bf69d73b02?t=1617348183776 [15.06.2021].

Johns Hopkins University: COVID-19 Dashboard by the Center for Systems Science and Engineering (CSSE) (Stand: 09.06.2021), in: https:/gisanddata.maps.arcgis.com/apps/opsdashboard/index.html\#/bda7594740fd40299423467b48e9ecf6 [09.06.2021].

Kongregation für die Glaubenslehre: Instruktion Dignitas Personae über einige Fragen der Bioethik (08.12.2008), in: https:/www.vatican.va/roman_curia/congrega tions/cfaith/documents/rc_con_cfaith_doc_20081208_dignitas-personae_ge.htm 1 [10.06.2021].

Kongregation für die Glaubenslehre: Note über die Moralität des Gebrauchs einiger Impfungen gegen Covid-19 (21.12.2020), in: https:/www.vatican.va/roman_ curia/congregations/cfaith/documents/rc_con_cfaith_doc_20201221_nota-vaccin i-anticovid_ge.html [10.06.2021].

Kummer, Susanne: Covid-19-Impfstoffe: Ethische Stellungnahme zu Fragen der Herstellung (12.02.2021), in: https:/www.imabe.org/imabeinfos/covid-19-impfst offe-ethische-stellungnahme-zu-fragen-der-herstellung\#_edn1 [10.06.2021].

Lintner, Martin M.: Das Triage-Problem in Italien während der COVID-19-Pandemie, in: Kröll, Wolfgang/Platzer, Johann/Ruckenbauer, Hans-Walter/Schaupp, Walter: Die Corona-Pandemie. Ethische, gesellschaftliche und theologische Reflexionen einer Krise, Baden-Baden: Nomos 2020 (=Bioethik in Wissenschaft und Gesellschaft, Band 10), 87-102.

Marini, Paolo: L'obbligo vaccinale per professionisti e operatori sanitari. Analisi dell'articolo 4 del Decreto Legge n. 44/2021 (09.04.2021), in: https:/www.altalex .com/documents/news/2021/04/09/obbligo-vaccinale-per-professionisti-e-operato ri-sanitari [15.06.2021].

Miscione, Michele: Conseguenze del rifiuto di vaccinazione anti Covid-19 dopo il D.L. 44/2021 (21.05.2021), in: https:/www.quotidianogiuridico.it/documents/20 21/05/21/conseguenze-del-rifiuto-di-vaccinazione-anti-covid-19-dopo-il-d-l-44-202 1 [15.06.2021].

Ministero della Salute: Piano vaccini anti COVID-19, in: https:/www.salute.gov.it/ portale/nuovocoronavirus/dettaglioContenutiNuovoCoronavirus.jsp?lingua=ital iano\&id=5452\&area=nuovoCoronavirus\&menu=vuoto [09.06.2021]. 
Ministero della Salute u. a.: Vaccinazione Anti SARS-CoV-s/Covid-19. Piano strategico. Elementi di preparazione e di implementazione della strategia vaccinale; in: Gazzetta Ufficiale della Repubblica Italiana, serie generale n. 72 (24.03.2021), 24-37, in: https:/www.trovanorme.salute.gov.it/norme/renderPdf.spring?serieg $\mathrm{u}=$ SG\&datagu=24/03/2021\&redaz=21 A01802\&artp=1\&art=1\&subart=1\&subart $1=10 \& v e r s=1 \&$ prog=001 [09.06.2020].

Ministero della Salute u. a.: Vaccinazione Anti SARS-CoV-2/COVID-19. Raccomandazioni ad interim sui gruppi target della vaccinazione anti-SARS-CoV-2/ COVID-19, in: Gazzetta Ufficiale della Repubblica Italiana, serie generale n. 72 (24.03.2021), 38-50, in: https:/www.trovanorme.salute.gov.it/norme/renderPdf. spring?seriegu=SG\&datagu=24/03/2021\&redaz=21A01802\&artp=1\&art=1\&suba $\mathrm{rt}=1 \&$ subart1=10\&vers=1\&prog=002 [09.06.2021] .

Our World in Data: Statistics and Research. Coronavirus (COVID-19) Deaths (Stand: 09.06.2021), in: https://ourworldindata.org/covid-deaths [09.06.2021].

Päpstliche Akademie für das Leben: Moralische Überlegungen zu Impfstoffen, für deren Produktion Zellen von abgetriebenen Föten verwendet werden (05.06.2005); Originalpublikation: Pontificia Accademia per la Vita: Riflessioni morali circa i vaccini preparati a partire da celluleprovenienti da feti umani abortiti, in: Medicina e Morale 2005/3, 618-628; dt. (nicht autorisierte) Übersetzung von Irmtraut Babel: Schriftenreihe der Aktion Leben e.V., Nr. 27, Absteinach 2007, in: https://www.horeb.org/fileadmin/eigene_dateien/Neue_Seite/ Downloads/PDF/PDFs/H-027.pdf [10.06.2021].

Pontificia Accademia per la Vita: Nota circa l'uso dei vaccini (31.07.2017), in: http://www.academyforlife.va/content/pav/it/the-academy/activity-academy/note -vaccini.html [10.06.2021].

Prentice, David: COVID-19 Vaccine Candidates and Abortion-Derived Cell Lines (30.09.2020; letztes Update: 02.06.2021), in: https://lozierinstitute.org/updatecovid-19-vaccine-candidates-and-abortion-derived-cell-lines/ [10.06.2021].

Rosenberger, Michael/Schaupp, Walter (Hg.): Ein Pakt mit dem Bösen? Die moraltheologische Lehre der „cooperatio ad malum“ und ihre Bedeutung heute (=Studien der Moraltheologie. Neue Folge 5), Münster: Aschendorff Verlag 2015.

Wadman, Meredith: Abortion opponents protest COVID-19 vaccines' use of fetal cells (05.06.2020), in: https:/www.sciencemag.org/news/2020/06/abortion-opponents-protest-covid-19-vaccines-use-fetal-cells [10.06.2021]. 
